\title{
Increased Color-Conversion Efficiency in Hybrid Light-Emitting Diodes utilizing Non-Radiative Energy Transfer
}

\author{
By Soontorn Chanyawadee, Pavlos G. Lagoudakis, * Richard T. Harley, \\ Martin D. B. Charlton, Dmitri V. Talapin, Hong Wen Huang, and \\ Chung-Hsiang Lin
}

There have been numerous efforts to increase the efficiency of solid-state lighting, light-emitting diodes (LEDs), and displays during the last decade. ${ }^{[1-3]}$ As the technologies for fabricating GaN-based LEDs and for synthesizing semiconductor colloidal nanocrystals (NQDs) mature, hybrid NQD-GaN LEDs are becoming promising candidates for highly efficient multicolor lighting. The high quantum yield and photostability of colloidal NQDs offer the possibility for flexible, low-cost, large-area, and simply processed optoelectronic devices, while their emission color can be tuned from the visible to the near-IR range of the electromagnetic spectrum by either changing their size or chemical composition. ${ }^{[4]}$ In addition, the epitaxial growth of $\mathrm{GaN}$ has now reached the stage where GaN-based LEDs have an internal quantum efficiency of $80 \% .^{[5]}$ Although their external quantum efficiency is inevitably limited by total internal reflection as a result of the high refractive-index contrast with air, several approaches to improve the outcoupling efficiency have been realized by implementing smart photonic-crystal and waveguide designs. ${ }^{[6,7]}$ Color-conversion LEDs consisting of colloidal NQD emitters pumped by GaN-based LEDs overcome the drawback of NQDs, i.e., low carrier transfer. ${ }^{[8]}$ A thin NQD layer deposited on an LED surface absorbs the high-energy photons that are electrically generated in the LED and subsequently re-emits lower-energy photons. As a result, there is no charge transfer among colloidal NQDs involved in this color-conversion process. However, the efficiency of radiative energy transfer is relatively low, $<10 \%$, because of several energy-loss steps in the transfer

[*] Prof. P. G. Lagoudakis, S. Chanyawadee, Prof. R. T. Harley School of Physics and Astronomy

University of Southampton

Southampton, SO17 1B) (UK)

E-mail: pavlos.lagoudakis@soton.ac.uk

Dr. M. D. B. Charlton

School of Electronics and Computer Science

University of Southampton

Southampton, SO17 1BJ (UK)

Prof. D. V. Talapin

Department of Chemistry, The University of Chicago

Chicago, IL 60637 (USA)

H. W. Huang, Dr. C.-H. Lin

Luxtaltek Corporation, Chunan

Miaoli 350, Taiwan (ROC)

DOI: 10.1002/adma.200902262 process, i.e., waveguided leaky mode losses, light-scattering from the NQDs, and the reduced efficiency of emission at blue wavelengths, absorption, and re-emission at longer wavelengths from the NQD layers.

A number of approaches to improve the efficiency of electrical carrier injection into NQD emitters are of interest. A blend of semiconducting polymers and NQDs, for instance, employs the comparatively better carrier-transfer properties of the semiconducting polymers to inject carriers into NQDs. ${ }^{[1,2]}$ Integration of NQD monolayers directly into a semiconductor $\mathrm{p}-\mathrm{n}$ junction diode possessing high carrier mobility was alternatively demonstrated. ${ }^{[9]}$ Nevertheless, the performance of this device is not comparable with that of conventional epitaxial semiconductor LEDs. The problems of charge imbalance at the emissive layer associated with different charge-transport efficiencies of the polymer transport layers and the difficulties in integrating NQDs into a semiconductor $\mathrm{p}-\mathrm{n}$ junction diode remain challenging. A new scheme of pumping NQDs by using non-radiative energy transfer has been demonstrated both theoretically ${ }^{[10,11]}$ and experimentally. ${ }^{[12,13]}$

Non-radiative energy transfer is a dipole-dipole interaction between donors and acceptors where the excitation energy of a donor is transferred to an acceptor. ${ }^{[14]}$ The non-radiative energy transfer rate $\left(k_{\mathrm{ET}}\right)$ scales linearly with spectral overlap and is proportional to $R^{-C}$, where $R$ is the donor-acceptor separation distance and $C$ is a constant. For example, $C=2$ or 6 describes layer-to-layer or point dipole-to-dipole energy transfer, respectively. ${ }^{[15,16]}$ In either case, to increase the energy-transfer rate and consequently color-conversion efficiency, the donor-acceptor separation distance has to be minimized. Hence, for the colloidal NQD/multiple quantum well (QW) LED configuration, the QW barrier and the top contact layer has to be as thin as possible, while remaining thick enough to minimize surface recombination of injected carriers and to allow for uniform spreading of the injected carriers over the active layers.

In this report, a novel method for utilizing non-radiative energy transfer in color-conversion lighting by depositing bright colloidal NQDs on surface-patterned GaN-based LEDs is proposed. Holes with elliptical cross-sections that penetrate through the active QW layers are fabricated on the LED surface, as shown in Figure 1a. This structure is referred to as a deep-etched LED. Unlike conventional color-conversion LEDs, this approach brings colloidal NQDs (acceptors) within close vicinity of the active layers (donors). The NQD absorption is tuned to overlap 

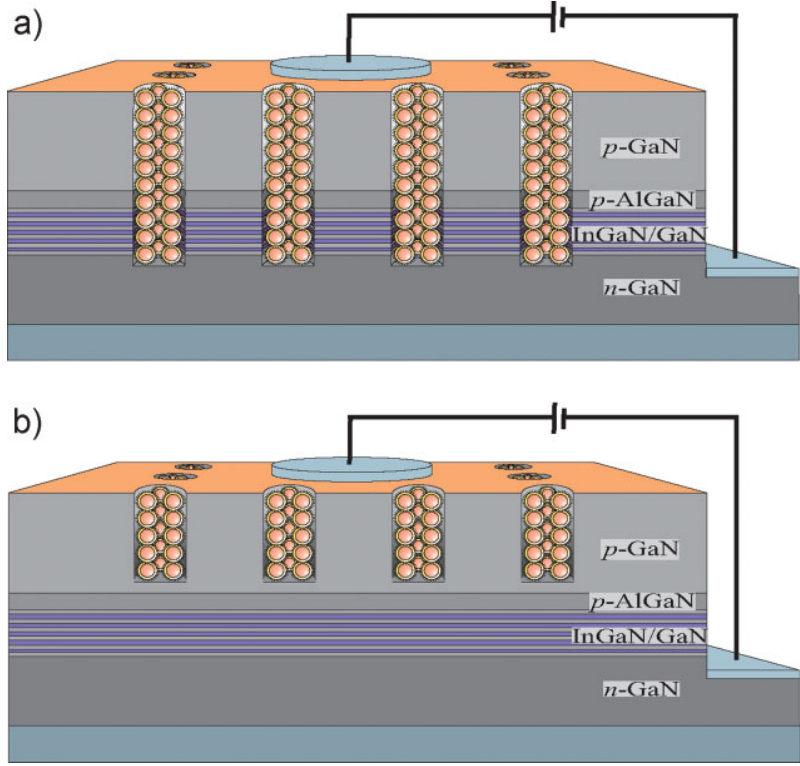

Figure 1. Schematic diagrams of a) the hybrid NQD/deep-etched LED and b) the hybrid NQD/shallow-etched LED (not to scale).

strongly with the LED emission and thus the two main requirements for efficient non-radiative energy transfer are satisfied. Electrically injected carriers in the active layers of the deep-etched LED can be transferred efficiently to the adjacent NQDs by means of non-radiative energy transfer. A control structure, where the patterning of the surface does not penetrate the active layers, is also fabricated as shown in Figure 1b, and is referred to as a shallow-etched LED. The donor-acceptor separation distance in the hybrid NQD/shallow-etched LED is large and so non-radiative energy transfer does not occur. By comparing the hybrid NQD/deep-etched LED with the hybrid NQD/shallow-etched LED, the hybrid NQD/deep-etched LED exhibits a twofold enhancement of NQD emission under the same emission intensity from the QWs.

Electroluminescence (EL) of the hybrid NQD/deep-etched LED devices was measured perpendicularly to the surface and compared to that of the control devices (shallow-etched LED). The EL intensity of the bare QW scales linearly with injection current and saturates at high injection current (not shown here). The linear increase of EL intensity implies that injected carriers form excitons in the QWs. The etching process partly removes active layers and inevitably introduces surface states to the side walls of the patterned structure. Consequently, the deep-etched LED emission is reduced in comparison to that of the control device for the same injection current. In Figure 2a, EL of the deep-etched LED at $7 \mathrm{~mA}$ (pink dotted line) is comparable with that of the shallow-etched LED at $3.8 \mathrm{~mA}$ (blue dotted line). The NQD emission pumped by the deep-etched LED is compared with NQD emission pumped by the shallow-etched LED to investigate the effect of non-radiative energy transfer on colorconversion efficiency. For the same EL of the bare QW emission $(460 \mathrm{~nm})$, NQD fluorescence pumped by the deep-etched LED (pink solid line) is significantly higher than NQD emission pumped by the shallow-etched LED (blue solid line). Colorconversion efficiency $\left(\eta_{c}\right)$ is defined as the ratio of the NQD
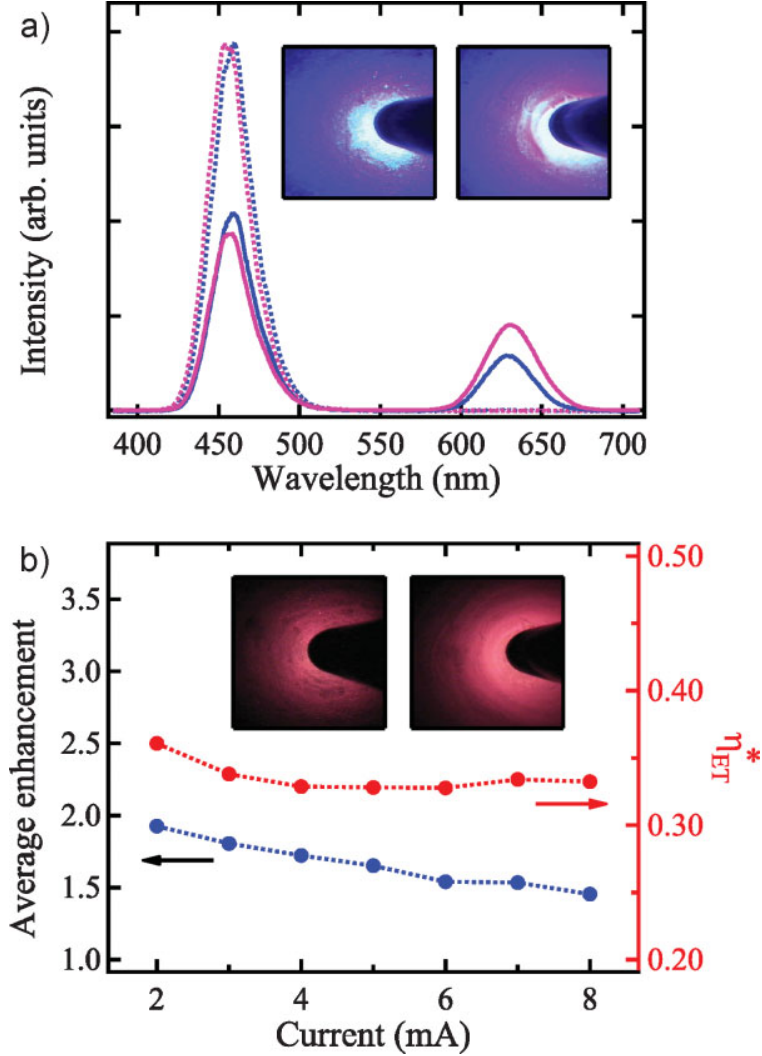

Figure 2. NQD emission enhancement and energy-transfer efficiency of the hybrid color-conversion LED. a) QW emission of the bare shallow-etched LED (dotted blue line) and the bare deep-etched LED (dotted pink line) at 3.8 and $7 \mathrm{~mA}$, respectively. The solid blue line (solid pink line) represents the corresponding EL of the hybrid NQD/shallow-etched LED (hybrid NQD/deep-etched LED). Inset: left (right) panel shows emission of the hybrid shallow etched (deep-etched) LED for injection currents that produce the same QW emission intensity in the absence of NQDs. b) Red solid circles represent effective non-radiative energy transfer efficiency versus the deep-etched LED injection current. Blue solid circles represent the enhancement of NQD emission. Inset: left (right) panel shows the emission of the NQDs only in the hybrid shallow-etched (deep-etched) LED.

emission intensity in the hybrid structure $\left(I_{\mathrm{NQD}}^{\mathrm{H}}\right)$ to the bare $\mathrm{QW}$ emission intensity $\left(I_{\mathrm{QW}}\right), \quad \eta_{\mathrm{C}}=I_{\mathrm{NQD}}^{\mathrm{H}} / I_{\mathrm{QW}} \cdot{ }^{[13]}$ The average color-conversion efficiency of the hybrid NQD/deep-etched LED in the linear regime is $20 \%$, i.e., a $43 \%$ increase from that of the hybrid NQD/shallow-etched LED.

We propose that the enhancement of NQD emission is caused by non-radiative energy transfer. Charge transfer through the insulating ligands of NQDs is inhibited because of the large band-offset between the carriers in the NQDs and surface molecules as well as between the NQDs and the QWs. ${ }^{[17]}$ Effective non-radiative energy transfer efficiency $\left(\eta_{\mathrm{ET}}^{*}\right)$ is estimated from $\eta_{\mathrm{ET}}^{*}=1-I_{\mathrm{QW}}^{\mathrm{H}} / I_{\mathrm{QW}}$, where $I_{\mathrm{QW}}^{\mathrm{H}}$ is the emission intensity of QW donors in the presence of NQD acceptors. Here, $\eta_{\mathrm{ET}}^{*}$ is averaged over the active area including both carriers that undergo non-radiative energy transfer and carriers that do not. At low injection current, $\eta_{\mathrm{ET}}^{*}$ decreases with increasing injection current and remains constant for injection current above ca. $4 \mathrm{~mA}$ as shown in Figure 2b. It is conceivable that, on increasing the injection current, heating and Coulomb screening lead to 
exciton dissociation into free electron-hole pairs, hence the decrease of $\eta_{\mathrm{ET}}^{*} \cdot{ }^{[1]}$ Figure $2 \mathrm{~b}$ shows average enhancement of NQD emission versus the injection current of the deep-etched LED, i.e., the ratio of NQD emission pumped by the deep-etched LED to that pumped by the shallow-etched LED for an injection current of the latter that produces the same QW emission intensity with the deep-etched LED in the bare configuration. Evidently, the NQD fluorescence is enhanced through the whole range of injection currents and slightly decreases with increasing injection current. Insets of Figure 2a show top-view images of the hybrid NQD/shallow-etched LED (left panel) and of the hybrid NQD/deep-etched LED (right panel), at injection currents that produce the same emission intensity at blue wavelengths from the shallow and deep-etched LEDs in the bare configuration (3.8 and $7 \mathrm{~mA}$, respectively). The corresponding images with a filter to cut the QW EL are shown in the inset of Figure 2b.

To verify the existence of non-radiative energy transfer in the hybrid NQD/deep-etched LED, the transient carrier dynamics in both the donor (QWs) and acceptor (NQDs) sites were investigated. The devices were excited at $400 \mathrm{~nm}$ with a $100 \mathrm{fs}$ pulse width and $250 \mathrm{kHz}$ repetition rate. The photoluminescence was coupled into a streak camera with 300 ps resolution. Time-resolved data of the shallow-etched LED and deep-etched LED are illustrated in Figure 3a,b, respectively. The inset of

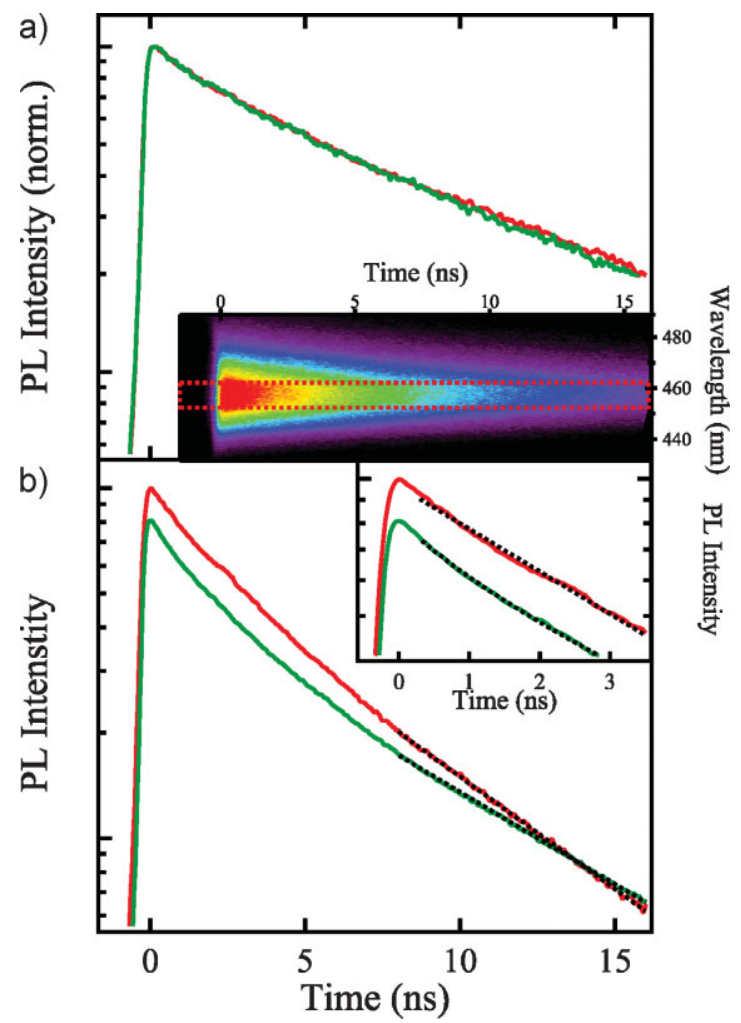

Figure 3. QW photoluminescence decay in the hybrid structures. a) QW emission decay of the shallow-etched LED without (red solid line) and with (green solid line) NQDs. Inset shows the streak image of the bare shallow-etched LED. b) QW emission decay of the deep-etched LED without (red solid line) and with (green solid line) NQDs. The dotted lines are the fits as described in text. Inset shows the effect of non-radiative energy transfer on the QW photoluminescence decay at early times.
Figure 3a shows a streak image and the energy window used to extract the QW dynamics. The carrier dynamics of the unetched QWs with (green solid line) and without (red solid line) deposited NQDs are virtually identical. These results suggest that in the NQD/shallow-etched LED where donor-acceptor distance is large, i.e., $100 \mathrm{~nm}$, non-radiative energy transfer does not occur. Hence, carriers in deposited NQDs are only generated from conventional radiative energy transfer. In contrast, carrier dynamics of the etched QWs change after depositing NQDs as shown in Figure 3b. The QW photoluminescence with deposited NQDs decays slightly faster over the first $4 \mathrm{~ns}$ and slower at later times compared to the bare QW. We interpret the faster decay as an effect of non-radiative energy transfer that introduces an additional decay channel for carriers in the QW. The slower decay at later times is believed to be an effect of surface passivation of the etched QWs from the organic ligands of the deposited NQDs. ${ }^{[18]}$

Since not all electron-hole pairs in the QWs undergo non-radiative energy transfer, the electron-hole pairs are categorized into two groups: the ones that undergo energy transfer and the ones that do not. As the non-radiative energy-transfer rate decreases dramatically with increasing donor-acceptor distance, electron-hole pairs close to the slots filled with NQDs (ca. $10 \mathrm{~nm}$ distance) can undergo non-radiative energy transfer, but the electron-hole pairs further away do not. The photoluminescence decay of the QW can be approximated with an exponential for the first $4 \mathrm{~ns}$ as shown in the inset of Figure $3 \mathrm{~b}$. To extract the actual non-radiative energy transfer rate $\left(k_{\mathrm{ET}}\right)$ and the percentage of electron-hole pairs that undergo non-radiative energy transfer, the photoluminescence decay of the QWs in the presence of the NQDs is approximated and fitted with the following equation

$I_{\mathrm{QW}}^{H}(t)=A e^{-k_{\mathrm{QW}} t}+B e^{-\left(k_{\mathrm{QW}}+k_{\mathrm{ET}}\right) t}$

where $A$ and $B$ are the fraction of electron-hole pairs that do not undergo and do undergo non-radiative energy transfer, respectively. $k_{\mathrm{QW}}$ is the total decay rate of the etched QWs, taking into account the effect of surface passivation. From the fitting, 18\% of generated electron-hole pairs experience non-radiative energy transfer with efficiency $\left(\eta_{\mathrm{ET}}=k_{\mathrm{ET}} /\left(k_{\mathrm{ET}}+k_{\mathrm{QW}}\right)\right)$ of $82 \%$. The energy -transfer efficiency is high because the donor-acceptor distance is limited only by the short ligand molecules on the surface of the NQDs, i.e., ca. $2 \mathrm{~nm}$. In previous studies of planar heterointerfaces, efficiencies as high as $65 \%$ were reported where a $2.5 \mathrm{~nm}$ thick capping layer was present to separate a single QW from NQDs. ${ }^{[19]}$ The absence of any barrier in this study allows for even higher non-radiative energy transfer efficiency to be observed.

Using scanning electron microscopy (SEM) images of the sample to obtain the total perimeter of the slots, it is estimated that non-radiative transfer occurs from only $2.3 \%$ of the LED surface, assuming the process occurs from a $10 \mathrm{~nm}$ thick layer in the QWs. Under uniform excitation of this structure, only ca. 3\% of all the generated excitons in the active layer would undergo energy transfer in a static picture, which is approximately one sixth of the value obtained from fitting the photoluminescence decay. However, exciton diffusion from regions further away from 
the holes towards the vicinity of the holes should increase the percentage of excitons undergoing non-radiative energy transfer. To investigate this possibility, a 2D Monte Carlo simulation was performed to calculate the percentage of excitons that undergo non-radiative energy transfer by taking into consideration exciton diffusion in the active layer of the deep-etched LED. In this model, the motion of excitons in the active region is dominated by their thermal energy and their momentum scattering probability; scattering with impurities or phonons is described by scattering time, $\tau_{\mathrm{s}}$, while the probability of radiative and non-radiative decay is given by $k_{\mathrm{QW}}$ and $k_{\mathrm{ET}}$, which was obtained from time-resolved measurements above. The momentum scattering time of carriers in InGaN QWs was estimated from $\tau_{\mathrm{s}}=m^{*} \mu / \mathrm{e}$, where $m^{*}$ is the effective mass, and $\mu$ is the carrier mobility. Although the carrier mobility in InGaN/GaN QWs varies widely in the literature ${ }^{[20,21]}$ for a momentum scattering time in the range of 0.01 to $1 \mathrm{ps}$ we obtain that ca. $18 \%$ of excitons undergo non-radiative energy transfer. This result is in reasonable agreement with the percentage obtained from fitting the photoluminescence decay with Equation (1) and therefore lends support to the interpretation.

Observation of non-radiative energy transfer from the donor site does not provide conclusive evidence that the energy is transferred to the desired acceptor sites, namely the NQDs in this case. Thus, it is imperative to prove the effect of non-radiative transfer on the transient NQD dynamics. Figure 4a,b show the fluorescence decay of NQD deposited on deep- and shallow-etched QW LEDs, respectively. It is apparent that the fluorescence of the former is higher because of non-radiative energy transfer. To exclude any quantitative effect that may originate from the larger number of NQDs in the patterned device, further investigation of the effect of non-radiative energy transfer on the NQD fluorescence decay was necessary. The normalized data of Figure 4a is subtracted from that of the normalized data of Figure $4 \mathrm{~b}$. Figure $4 \mathrm{c}$ shows that the energy transfer creates additional carriers in the deposited NQDs at an early time as shown by the blue area (positive counts). A $20 \mathrm{~nm}$ window centered at the NQD fluorescence peak, as depicted with dashed lines in Figure 4c, was used to extract the average carrier dynamics of the deposited NQDs shown in Figure 4d. The carrier dynamics of the deposited NQDs, including non-radiative energy transfer from the QWs, can be described by Equation $2^{[22]}$

$I_{\mathrm{NQD}}^{\mathrm{H}}(t) \propto \frac{k_{\mathrm{ET}}}{k_{\mathrm{NQD}}-k_{\mathrm{QW}}-k_{\mathrm{ET}}}\left(e^{-\left(k_{\mathrm{QW}}+k_{\mathrm{ET}}\right) t}-e^{-k_{\mathrm{NQD}} t}\right)$

where $k_{\mathrm{NQD}}$ is the NQD fluorescence decay rate. By using the parameters obtained from the measurement of the time-resolved QW dynamics, the fitted dynamics caused by energy transfer from the QWs to the NQDs, as shown by the dotted line in Figure $4 \mathrm{~d}$, is in good agreement with experiment (solid red line), unequivocally demonstrating non-radiative energy transfer from the deep-etched LED to the NQDs.

In conclusion, a color-conversion LED consisting of a surface-patterned blue-light-emitting LED and colloidal NQDs that promotes efficient non-radiative energy transfer from a higher-energy epitaxial light source to lower-energy emission

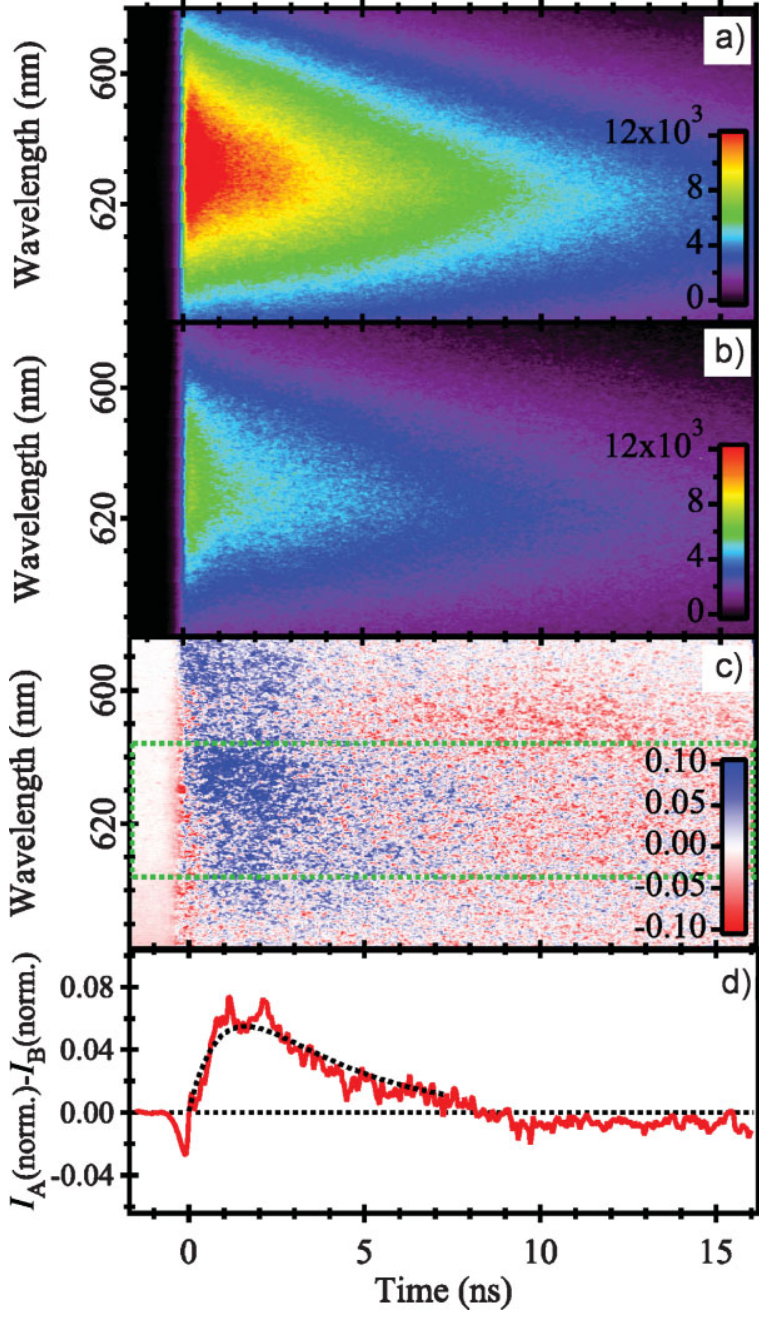

Figure 4. Spectrally and time-resolved NQD fluorescence decay in the hybrid structures. a) NQDs on the deep-etched LED. b) NQDs on the shallow-etched LED. c) Difference of fluorescence dynamics calculated from the normalized data of $(a, b)$. d) The average difference of fluorescence dynamics extracted from $20 \mathrm{~nm}$ energy window centered at the peak of the NQD emission. The dotted line is a fit as described in text.

fluorophores has been proposed. A twofold enhancement of the colloidal NQD emission is demonstrated and attributed to non-radiative energy transfer by studying the transient dynamics both at donor and acceptor sites. This new design introduces a high-performance color-conversion LED with applications in solid-state lighting, displays, and lasers from fluorophores of low carrier mobility.

\section{Experimental}

A schematic diagram of the hybrid NQD/deep-etched LEDs is shown in Figure 1a. A $2 \mu \mathrm{m}$ thick undoped $\mathrm{GaN}$ buffer layer was grown on a sapphire substrate followed by a $2 \mu \mathrm{m}$ thick $n$-doped $\mathrm{GaN}$. The active layer consisted of five InGaN/GaN QWs and a $50 \mathrm{~nm}$ thick p-doped AlGaN layer was grown on top. The device was terminated with a $0.2 \mu \mathrm{m}$ thick p-doped GaN layer. 


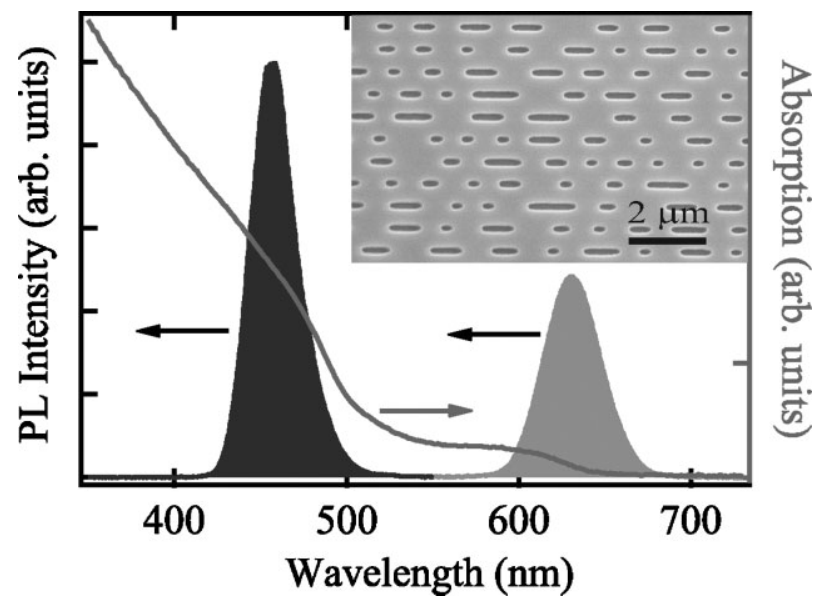

Figure 5. Spectral overlap of the QW emission (blue) centered at $460 \mathrm{~nm}$ and the NQD absorption (red solid line). NQD emission peak (orange) is centered at $630 \mathrm{~nm}$ at room temperature. Inset shows an SEM image of the shallow-etched LED surface.

An array of $450 \mathrm{~nm}$ deep slots with elliptical cross-sections was etched through the top layers, penetrating the multiple QWs. Figure $1 \mathrm{~b}$ presents a schematic diagram of the hybrid NQD/shallow-etched LED where an array of $150 \mathrm{~nm}$ deep slots were fabricated on the LED. The array patterns of both deep-etched and shallow-etched LEDs are identical. The inset of Figure 5 shows an SEM image of the shallow-etched LED surface. The array forms a photonic structure designed to improve the emission from the LED; its details are not relevant to this experiment as the same design has been used for both deep- and shallow-etched LEDs. The photoluminescence peak of the LED is at $460 \mathrm{~nm}$ at room temperature as shown in Figure 5. $\mathrm{CdSe} / \mathrm{CdS}$ rod-shaped core/shell NQDs capped with hexadecylamine, trioctylphosphine oxide (TOPO), and tri-n-octylphosphine (TOP) were used as color converters in this study [23]. The absorption and fluorescence spectra of the colloidal NQDs are illustrated in Figure. 5. The aspect ratio (length/diameter) of the NQDs is 3.6. The NQDs were dispersed in chloroform. The hybrid NQD/deep-etched LED and the hybrid NQD/ shallow-etched LED were prepared by drop-casting the solution of colloidal NQDs onto the corresponding LEDs. The hybrid LED was characterized after complete evaporation of the solvent under ambient atmosphere (ca. $10 \mathrm{~min}$ ).

Nanoimprinting lithography was used to fabricate patterns onto LEDs. A $50 \mathrm{~nm}$ thick $\mathrm{SiO}_{2}$ layer was initially deposited onto the LEDs by plasma-enhanced chemical vapor deposition (PECVD) followed by a spin-coated polymer layer. A mold was brought into contact with the polymer layer under certain pressure. By applying heat above the glass-transition temperature of the polymer, the pattern was transferred from the mold to the polymer layer. The LED samples and the mold were then cooled to room temperature to release the mold. Reactive-ion etching (RIE) with $\mathrm{CF}_{4}$ plasma was used to remove the residual polymer layer and transfer the pattern onto $\mathrm{SiO}_{2}$.

The patterns of different depths were eventually transferred to the LEDs using inductively coupled plasma RIE (ICP-RIE). $\mathrm{Cl}_{2}$ and $\mathrm{Ar}$ etching gases were introduced into the reactor chamber through independent electronic mass-flow controllers (MFCs) that can control the flow rate with an accuracy of about $1 \mathrm{sccm}$. An automatic pressure controller (APC) was placed near the exhaust of the chamber to control the chamber pressure. The ICP etching rate was determined to be ca. $7.5 \mathrm{~nm} \mathrm{~s}^{-1}$ associated with the following conditions: the flow rate ratio $\mathrm{Cl}_{2} / \mathrm{Ar}=10 / 25$ with the ICP source power, bias power set at $200 / 200 \mathrm{~W}$, and chamber pressure of 2.5 mTorr. ( $1 \mathrm{Torr}=133.32 \mathrm{~Pa}$ ) The residual $\mathrm{SiO}_{2}$ layer was subsequently removed by a buffer oxidation etchant (BOE).

Received: July 7, 2009

Published online: November 6, 2009

[1] V. L. Colvin, M. C. Schlamp, A. P. Alivisatos, Nature 1994, 370, 354.

[2] S. Coe, W. K. Woo, M. Bawendi, V. Bulovic, Nature 2002, 420, 800.

[3] J. M. Caruge, J. E. Halpert, V. Wood, V. Bulovic, M. G. Bawendi, Nat. Photonics 2008, 2, 247.

[4] Q. Sun, Y. A. Wang, L. S. Li, D. Y. Wang, T. Zhu, J. Xu, C. H. Yang, Y. F. Li, Nat. Photonics 2007, 1, 717.

[5] T. Nishida, H. Saito, N. Kobayashi, Appl. Phys. Lett. 2001, 79, 711.

[6] H. Y. Ryu, Y. H. Lee, R. L. Sellin, D. Bimberg, Appl. Phys. Lett. 2001, 79, 3573.

[7] J. Y. Kim, M. K. Kwon, K. S. Lee, S. J. Park, S. H. Kim, K. D. Lee, Appl. Phys. Lett. 2007, 91, 3.

[8] H. S. Chen, S. J. J. Wang, C. J. Lo, J. Y. Chi, Appl. Phys. Lett. 2005, 86, 131 13905.

[9] A. H. Mueller, M. A. Petruska, M. Achermann, D. J. Werder, E. A. Akhadov, D. D. Koleske, M. A. Hoffbauer, V. I. Klimov, Nano Lett. 2005, 5, 1039.

[10] V. M. Agranovich, D. M. Basko, C. C. La Rocca, F. Bassani, J. Phys.: Condens. Matter 1998, 10, 9369.

[11] D. Basko, G. C. La Rocca, F. Bassani, V. M. Agranovich, Eur. Phys. J, B 1999, 8, 353.

[12] M. Achermann, M. A. Petruska, S. Kos, D. L. Smith, D. D. Koleske, V. I. Klimov, Nature 2004, 429, 642

[13] M. Achermann, M. A. Petruska, D. D. Koleske, M. H. Crawford, V. I. Klimov, Nano Lett. 2006, 6, 1396.

[14] T. Förster, Ann. Phys. 1948, 437, 55.

[15] H. Kuhn, J. Chem. Phys. 1970, 53, 101.

[16] J. Hill, S. Y. Heriot, O. Worsfold, T. H. Richardson, A. M. Fox, D. D. C. Bradley, Phys. Rev. B 2004, 69, 04104303.

[17] P. O. Anikeeva, C. F. Madigan, J. E. Halpert, M. G. Bawendi, V. Bulovic, Phys. Rev. B 2008, 78, 08508434.

[18] S. Chanyawadee, R. T. Harley, M. Henini, D. V. Talapin, P. G. Lagoudakis, Phys. Rev. Lett. 2009, 102, 07707402.

[19] S. Rohrmoser, J. Baldauf, R. T. Harley, P. G. Lagoudakis, S. Sapra, A. Eychmuller, I. M. Watson, Appl. Phys. Lett. 2007, 91, 09209126.

[20] T. Wang, H. Saeki, J. Bai, T. Shirahama, M. Lachab, S. Sakai, P. Eliseev, Appl. Phys. Lett. 2000, 76, 1737

[21] C.-A. Chang, C.-F. Shih, N.-C. Chen, T. Y. Lin, K.-S. Liu, Appl. Phys. Lett. 2004, 85, 6131.

[22] S. Chanyawadee, P. G. Lagoudakis, R. T. Harley, D. G. Lidzey, M. Henini, Phys. Rev. B 2008, 77, 19319402.

[23] D. V. Talapin, R. Koeppe, S. Gotzinger, A. Kornowski, J. M. Lupton, A. L. Rogach, O. Benson, J. Feldmann, H. Weller, Nano Lett. 2003, 3, 1677. 